men cells explanted from serially transplanted mouse mammary tumours, which during the serial transplantations have lost the capacity to develop in situ an acinar organization and do not in situ liberate virus, fail to form domes and also do not support detectable replication of mouse mammary tumour virus. In short, the capacity of murine mammary epithelial cells to support replication of mammary tumour virus is positively correlated with the cells' capacity to assume an acinar organization.

Cells which can form acini and domes, irrespective of whether or not they are infected and "transformed" by mammary tumour virus and are supporting its replication, respond in culture to contact inhibition of multiplication. By contrast the serially transplanted mammary tumour cells, which fail to form acini or domes and do not produce virus, behave as do transformed fibroblasts, overgrowing each other, continuing to mitosis and reaching high saturation densities. In short, transformation of mammary epithelial cells does not apparently immediately result in loss of contact inhibition. As McGrath et al. comment, this fact may in part explain why attempts to detect transformation in vitro with the mouse mammary tumour viruses have universally failed.

The growth of primary mouse mammary tumours, they argue, probably results from a failure of growth control at the tissue rather than the cellular level of organization, the difference between normal and tumour tissues lying in the number of acini that develop rather than the number of mitoses of any particular cell. McGrath et al. suggest that in primary tumours the progenitor cells of acini are apparently unable to recognize "spacing regulations imposed on acinar growth which normally fix an upper limit to the number of acini which can occupy a given area". If that is the case it will indeed be difficult to devise an in vitro biological assay for mammary tumour viruses, but it would be interesting to test whether or not the cells in domes or cultures of mammary epithelial cells from uninfected healthy mice can be successfully infected by exogenous mammary tumour virus, and then support virus replication, as do the domes in primary cultures of cells from infected animals.

\section{MACHINE PERCEPTION Patferns and Pictures}

from a Correspondent

A CONFERENCE on machine perception of patterns and pictures was organized by the Institute of Physics' Physics in Industry Subcommittee in collaboration with the Institution of Electrical Engineers and the National Physical Labora- tory at Teddington from April 12 to 14.

Most of the contributions assumed as their framework the standard four-stage paradigm of pattern recognition. First, the visual or auditory signal to be recognized is measured. In the preprocessing stage, various operators are applied uniformly to the signal to reduce its complexity. In stage three, features are extracted from the signal that will, it is hoped, be crucial in classifying the input. The final stage takes a representation of the input as a point in multidimensional feature space and classifies it by various linear, piecewise linear or non-linear decision techniques. The standard video measuring devices are flying spot scanners, television cameras or solid state arrays with the hope of electronically-deflected laser scanning in the near future. The recognition logic is usually designed on a computer but implemented in hardwired form.

$\mathrm{Mr}$ M. Nadler (Honeywell-Bull, France) reviewed the optical character recognition technology which is available and concluded that although machines for reading specially designed fonts at high speed are now being installed, replacing many key punchers, their high cost is preventing wider use. Two contributions described the Post Office's use of orthodox pattern recognition techniques to read machine printed post codes but nobody has yet designed a satisfactory system for handwritten material. Dr J. R. Ullman (National Physical Laboratory) suggested that the fault may lie in a paradigm that does not adequately express relationships of dependency between the features.

In a review of biomedical applications, Dr D. Rutovitz (MRC Cytogenetics Unit, London) attacked the academic tendency to conduct idealized computer experiments and emphasized the importance of good preparation and automatic presentation of specimens for computer analysis. Automatic chromosome analysis is now competitive with human analysis but many other possible applications, such as the analysis of fibrous sections, are far from being realized.

$\mathrm{Mr}$ F. Billingsley (Jet Propulsion Laboratory, Pasadena), who is responsible for some of the consciousnessexpanding photographs from the National Aeronautics and Space Administration, discussed picture processing in the space programme. This work in

\title{
Effect of Anti-kappa Antisera on B Cells
}

THE search for chemical or biological agents which act specifically against $\mathbf{B}$ or $\mathrm{T}$ lymphocytes proceeds apace. The early studies of Reif and later of Raff and their colleagues have successfully established the $\theta$-antigen as a characteristic of $T$ cells and anti- $\theta$ antisera as the means by which destructive elimination of $T$ cells from a given cell population can be achieved. The presence of complement receptors has been proposed as an attribute of $B$ cells by Nussenzweig and his colleagues and indeed the possession of surface bound immunoglobulins is widely accepted as a $\mathbf{B}$ cell rather than a $\mathbf{T}$ cell characteristic.

Miller and his colleagues accepted this character as a starting point, and in next Wednesday's Nature New Biology (May 3) they describe experiments in which the light chain component of the surface immunoglobulins of B cells is attacked by heterologous anti-kappa antisera.

Ninety-five per cent of the light chains of mouse immunoglobulins are of kappa type, and Miller et al. prepared a series of rabbit anti-mouse kappa antisera using Bence Jones proteins from various mouse myelomas as their immunogens. The antisera, after absorption with mouse thymocytes, were tested against various kinds of mouse thoracic duct cells; first, normal mouse cells; second, cells from nude mice (as a source of B cells); and, third, cells from irradiated $\mathbf{F}_{1}$ mice that had, four days previously, received an intravenous injection of parental cells. In earlier experiments it was shown that these last cells are a pure $\mathrm{T}$ cell population. The anti-kappa antiserum killed a small proportion of cells from normal mouse thoracic duct lymph, nearly all the cells from nude mice and very few of the cells from the hybrid animals. The studies were carried out in conjunction with other tests for $T$ cells (anti- $\theta$ ) and $B$ cells (the capacity to bind immune complexes). The results of all three tests were very similar, and Miller and his associates conclude that anti-kappa antisera are useful diagnostic and selective agents for B cells.

Not all Miller et al.'s anti-kappa sera, however, were of the same potency (the same is true of anti- $\theta$ ). Nevertheless, there was a rough correlation between anti-kappa binding capacities of the various cells tested after incubation with anti-kappa sera and, although in the main B cell activity was depleted in various transfer systems, it proved impossible to deplete spleen cell populations of the capacity to respond to sheep erythrocytes. Miller et al. reject the possibility that B cells in the spleen do not have attackable surface immunoglobulins and conclude that $\mathbf{B}$ cell activity can be generated from stem cells in the transferred spleens which are not susceptible to the cytotoxic effects of anti-kappa antisera. 